\title{
Sur le minimum de la fonction de Brjuno
}

\author{
Michel Balazard et Bruno Martin
}

12 février 2020

\begin{abstract}
The Brjuno function attains a strict global minimum at the golden section.
\end{abstract}

\section{KEYWORDS}

Gauss transformation, Brjuno function, Golden section

MSC classification : 26D07, 11A55

La transformation de Gauss,

$$
\alpha(x)=\{1 / x\},
$$

où $\{t\}$ désigne la partie fractionnaire du nombre réel $t$, est bien définie sur l'ensemble

$$
X=] 0,1[\backslash \mathbb{Q},
$$

et à valeurs dans $X$. On peut donc considérer ses itérées successives, définies par les relations $\alpha_{0}(x)=x$ et $\alpha_{k+1}(x)=\alpha_{k}(\alpha(x))$ pour $k \geqslant 0$, et les produits

$$
\beta_{k}(x)=\prod_{j=0}^{k} \alpha_{j}(x) \quad(k \in \mathbb{N}, x \in X),
$$

avec la convention supplémentaire $\beta_{-1}(x)=1$. Comme $\alpha$ est continue sur $X$, les $\alpha_{k}$ et les $\beta_{k}$ le sont également.

La fonction de Brjuno est alors définie, pour tout $x \in X$, comme la somme, éventuellement égale à $+\infty$, de la série à termes positifs

$$
\Phi(x)=\sum_{k \geqslant 0} \beta_{k-1}(x) \ln \left(1 / \alpha_{k}(x)\right) .
$$

Les points de convergence sont appelés nombres de Brjuno; nous noterons $\mathcal{B}$ leur ensemble. La fonction et les nombres de Brjuno interviennent dans la théorie des systèmes dynamiques (cf. par exemple [2], [3], [4, [6]). L'ensemble $\mathcal{B}$ est de mesure 1 , et donc dense dans $[0,1]$. 
L'ensemble $\mathcal{B}$ des nombres de Brjuno est stable par $\alpha$. La fonction $\Phi$ vérifie l'équation fonctionnelle

$$
\Phi(x)=\ln (1 / x)+x \Phi(\alpha(x)) \quad(x \in \mathcal{B}),
$$

et, plus généralement,

$$
\Phi(x)=\Phi_{K}(x)+\beta_{K}(x) \Phi\left(\alpha_{K+1}(x)\right) \quad(K \in \mathbb{N}, x \in \mathcal{B})
$$

où $\Phi_{K}$ désigne la somme partielle

$$
\Phi_{K}(x)=\sum_{k=0}^{K} \beta_{k-1}(x) \ln \left(1 / \alpha_{k}(x)\right) .
$$

Les fonctions $\Phi_{K}$ sont définies et continues sur $X$.

Dans l'article [5], Rivoal émet plusieurs conjectures sur les valeurs extrémales de séries diophantiennes, dont certaines sont proches de la fonction de Brjuno. Le théorème suivant fournit la réponse à une question posée aux auteurs par Rivoal, concernant la fonction $\Phi$ elle-même.

Théorème Soit $\theta=(\sqrt{5}-1) / 2=0,618$... le nombre d'or. Pour tout nombre de Brjuno $x \neq \theta$, on $a \Phi(x)>\Phi(\theta)$.

La démonstration de ce théorème s'appuie sur cinq propositions auxiliaires.

Proposition 1 Soit $r$ un nombre rationnel, élément du segment $[0,1]$. On a alors

$$
\Phi(x) \rightarrow \infty \quad(x \rightarrow r, x \in \mathcal{B}) .
$$

\section{Démonstration}

On a $\Phi(x) \geqslant \Phi_{0}(x)=\ln 1 / x$, donc le résultat est vrai si $r=0$. On a

$$
\Phi(x)=\ln 1 / x+x \Phi(\alpha(x)) \geqslant \frac{1}{2} \Phi(1 / x-1) \quad(1 / 2<x<1),
$$

donc le résultat est aussi vrai si $r=1$.

Si $r$ est un nombre rationnel de ]0,1[, écrivons $r$ sous forme d'une fraction continue finie,

$$
r=\left[0 ; a_{1}, \ldots, a_{k}\right] \quad\left(k \geqslant 1, a_{1}, \ldots, a_{k} \in \mathbb{N}^{*}, a_{k} \geqslant 2\right) .
$$

L'application $\varphi: t \mapsto\left[0 ; a_{1}, \ldots, a_{k-1}, a_{k}+t\right]$ est un homéomorphisme de ] - 1, 1[ sur un certain voisinage de $r$ dans $] 0,1[$.

Pour $t \in X$, ce qui entraîne $\varphi( \pm t) \in X$, on a

$$
\alpha_{k}(\varphi(t))=t \quad ; \quad \alpha_{k}(\varphi(-t))=1-t,
$$


et $\beta_{k-1}(\varphi( \pm t))$ est minorée par une constante positive (dépendant de $r$, cf. [1], (18)-(19), p. 199) pour $t \in X$. Comme

$$
\Phi(\varphi(t)) \geqslant \beta_{k-1}(\varphi(t)) \Phi\left(\alpha_{k}(\varphi(t))\right) \quad(t \in]-1,1[\backslash \mathbb{Q}),
$$

le résultat général découle des cas particuliers $r=0,1$.

Posons maintenant

$$
C=\inf _{x \in \mathcal{B}} \Phi(x) .
$$

On a $0 \leqslant C<\infty$, car $\Phi$ est à valeurs $\geqslant 0$ et $\mathcal{B}$ n'est pas vide.

Proposition 2 La borne inférieure $C$ est le minimum de la fonction de Brjuno sur $\mathcal{B}$ : il existe $r \in \mathcal{B}$ tel que $C=\Phi(r)$.

\section{Démonstration}

Soit $\left(x_{n}\right)_{n \geqslant 1}$ une suite d'éléments de $\mathcal{B}$ telle que

$$
\Phi\left(x_{n}\right) \rightarrow C \quad(n \rightarrow \infty) .
$$

En remplaçant éventuellement cette suite par une de ses sous-suites, nous pouvons supposer que la suite $\left(x_{n}\right)$ est elle-même convergente, vers une limite $r \in[0,1]$. La proposition 1 et l'hypothèse de convergence vers $C$ de la suite $\Phi\left(x_{n}\right)$ entraînent alors que $r$ est irrationnel. Nous allons montrer que $r \in \mathcal{B}$ et que $\Phi(r)=C$.

Soit $K \in \mathbb{N}$. Par définition de $\Phi$ on a $\Phi\left(x_{n}\right) \geqslant \Phi_{K}\left(x_{n}\right)$ pour tout $n$. La continuité de $\Phi_{K}$ en tout point irrationnel entraîne donc, par passage à la limite, l'inégalité $C \geqslant \Phi_{K}(r)$. Comme $K$ est arbitraire, cela prouve que $r$ est un nombre de Brjuno, et que $C \geqslant \Phi(r)$. Comme on a aussi, par définition de $C$, l'inégalité inverse $\Phi(r) \geqslant C$, on en déduit l'égalité $C=\Phi(r)$. Cette borne inférieure est donc bien un minimum.

Proposition 3 Soit $r \in \mathcal{B}$ tel que $C=\Phi(r)$. Pour tout $K \in \mathbb{N}$, on a

$$
C=\Phi(r) \geqslant \frac{\Phi_{K}(r)}{1-\beta_{K}(r)}
$$

\section{Démonstration}

En appliquant la relation (2) à $x=r$, nous obtenons

$$
C=\Phi(r)=\Phi_{K}(r)+\beta_{K}(r) \Phi\left(\alpha_{K+1}(r)\right) \geqslant \Phi_{K}(r)+C \beta_{K}(r),
$$

par définition de $C$. L'assertion en résulte. 
Proposition 4 Pour tout $K \in \mathbb{N}$, on a

$$
\Phi(\theta)=\frac{\Phi_{K}(\theta)}{1-\beta_{K}(\theta)}
$$

\section{Démonstration}

En effet, en appliquant la relation (2) à $x=\theta$, nous obtenons

$$
\Phi(\theta)=\Phi_{K}(\theta)+\beta_{K}(\theta) \Phi\left(\alpha_{K+1}(\theta)\right)=\Phi_{K}(\theta)+\beta_{K}(\theta) \Phi(\theta),
$$

puisque $\theta$ est point fixe de $\alpha$.

Proposition 5 Soit $r \in \mathcal{B}$ tel que $C=\Phi(r)$. On a $r \geqslant \theta$.

\section{Démonstration}

D'après les propositions 3 et 4 avec $K=0$, et la définition de $C$, on a

$$
\frac{\Phi_{0}(\theta)}{1-\beta_{0}(\theta)}=\Phi(\theta) \geqslant C=\Phi(r) \geqslant \frac{\Phi_{0}(r)}{1-\beta_{0}(r)} .
$$

Or, pour $0<x<1$,

$$
\frac{\Phi_{0}(x)}{1-\beta_{0}(x)}=\frac{\ln 1 / x}{1-x}=\int_{0}^{1} \frac{d t}{(1-t) x+t},
$$

est une fonction strictement décroissante de $x$, donc $\theta \leqslant r$.

$\mathrm{Au}$ vu de la proposition 2, l'énoncé suivant est équivalent à celui du théorème.

Proposition 6 Soit $r \in \mathcal{B}$ tel que $C=\Phi(r)$. On a $r=\theta$.

\section{Démonstration}

D'après les propositions 3 et 4 avec $K=1$, et la définition de $C$, on a

$$
\frac{\Phi_{1}(\theta)}{1-\beta_{1}(\theta)}=\Phi(\theta) \geqslant C=\Phi(r) \geqslant \frac{\Phi_{1}(r)}{1-\beta_{1}(r)} .
$$

Pour $1 / 2<x<1$, posons

$$
f(x)=\frac{\Phi_{1}(x)}{1-\beta_{1}(x)}=\frac{\ln 1 / x+x \ln 1 / \alpha(x)}{1-(1-x)}=\frac{\ln 1 / x}{x}+\ln \frac{x}{1-x},
$$

puisque $\alpha(x)=(1-x) / x$ pour $1 / 2<x<1$.

On a

$$
f^{\prime}(x)=\frac{\ln x}{x^{2}}+\frac{2 x-1}{x^{2}(1-x)},
$$


fonction qui a le signe de

$$
g(x)=2 x-1+(1-x) \ln x .
$$

La fonction $g$ est strictement croissante sur $] 0,1]$ et $g(0,61)=0,027 \ldots>0$. Par conséquent, la fonction $f$ est strictement croissante sur $[\theta, 1[$. La relation (3) et la proposition 5 entraînent donc l'égalité $r=\theta$.

Pour mettre en évidence la substance de la démonstration que nous venons d'exposer, remplaçons dans la définition de la fonction de Brjuno, la fonction logarithme par une fonction arbitraire $u:[1, \infty[\rightarrow[0, \infty[$, et posons

$$
\Psi_{u}(x)=\sum_{k \geqslant 0} \beta_{k-1}(x) u\left(1 / \alpha_{k}(x)\right)
$$

(cf. 4], §3.2, p. 609). Comme tout irrationnel quadratique appartient à l'ensemble $\mathcal{B}_{u}$ des points de convergence de cette série*, cet ensemble est toujours dense. L'équation fonctionnelle (11) devient

$$
\Psi_{u}(x)=u(1 / x)+x \Psi_{u}(\alpha(x)) \quad\left(x \in \mathcal{B}_{u}\right),
$$

En toute généralité, il n'est pas vrai que la fonction $\Psi_{u}$ atteigne un minimum global au nombre d'or. En effet, si $u(t)=t^{a}$, avec $a<1$, et $\theta^{\prime}=1-\theta=1 /(2+\theta)$, de sorte que $\alpha\left(\theta^{\prime}\right)=\theta$, on a

$$
\Psi_{u}(\theta)=\theta^{-a}+\theta \Psi_{u}(\theta)=\theta^{-a}+\left(1-\theta^{\prime}\right) \Psi_{u}(\theta),
$$

donc $\theta^{\prime} \Psi_{u}(\theta)=\theta^{-a}$, et

$$
\Psi_{u}\left(\theta^{\prime}\right)=\theta^{\prime-a}+\theta^{\prime} \Psi_{u}(\theta)=\theta^{\prime-a}+\theta^{-a}<\theta^{-a} / \theta^{\prime}=\Psi_{u}(\theta),
$$

car la dernière inégalité équivaut à $\theta^{1-a}<\theta^{1-a}$, c'est-à-dire à $\theta^{\prime}<\theta$, inégalité vraie.

En remplaçant, dans les énoncés, $\Phi$ par $\Psi_{u}, \mathcal{B}$ par $\mathcal{B}_{u}$, et $\Phi_{K}$ par la somme partielle de $\Psi_{u}$ correspondante, examinons les conditions de validité des six propositions précédentes.

- La proposition 1 reste valable si, et seulement si

$$
u(x) \rightarrow \infty \quad(x \rightarrow \infty) .
$$

- Sous l'hypothèse (5), la proposition 2 reste valable si

la fonction $u$ est continue en chaque point irrationnel.

- Sous les hypothèses (5) et (6), les propositions 3 et 4 restent valables.

*. D'une part, on a l'inégalité $\beta_{k-1} \leqslant 1 / F_{k+1}$, où $F_{n}$ désigne le $n^{\mathrm{e}}$ nombre de Fibonacci ; d'autre part, lorsque $x$ est un irrationnel quadratique, l'ensemble des valeurs $\alpha_{k}(x)(k \in \mathbb{N})$, est fini. 
- Sous les hypothèses (5) et (6), la proposition 5 reste valable si

$$
\text { la fonction } \left.x \mapsto \frac{u(1 / x)}{1-x} \text { est strictement décroissante sur }\right] 0,1[\text {. }
$$

- Sous les hypothèses (15), (6) et (7), la proposition [6 reste valable si

$$
\text { la fonction } x \mapsto \frac{u(1 / x)}{x}+u\left(\frac{x}{1-x}\right) \text { est strictement croissante sur }[\theta, 1[\text {. }
$$

Notons que l'hypothèse (7) entraîne que $u(x)$ tend vers 0 quand $x$ tend vers 1 . La proposition suivante décrit une classe de fonctions (comprenant la fonction logarithme) vérifiant les hypothèses (5), (6), (7) et (8) ; les fonctions $\Psi_{u}$ correspondantes ont donc toutes un minimum global strict au nombre d'or.

Proposition 7 Soit $u:[1, \infty[\rightarrow[0, \infty[$ une fonction telle que

- $u(1)=0$;

- $u(x) \rightarrow \infty \quad(x \rightarrow \infty)$;

- la fonction $v$ définie par $v(t)=t u(t)$ est strictement convexe sur $[1, \infty[$.

Alors les hypothèses (5), (6) et (7) sont vérifiées. Si, de plus,

- la fonction $x \mapsto u(x /(1-x))$ est convexe sur $[1 / 2,1[$;

- $u^{\prime}(1 / \theta) \geqslant u(1 / \theta)$

(où $u^{\prime}$ désigne la dérivée à droite), alors l'hypothèse (8) est vérifiée.

\section{Démonstration}

La convexité de $v$ entraîne sa continuité, donc aussi celle de $u$, sur ]1, $\infty[$. Les deux conditions $u \geqslant 0$ et $u(1)=0$ entraînent aussi la continuité à droite au point 1 .

La stricte convexité de $v$ et le fait que $v(1)=0$ entraînent que la pente $w(t)=v(t) /(t-1)$ est une fonction strictement croissante de $t>1$. Par conséquent la fonction $v$ est elle-même strictement croissante, ainsi que la fonction $u(t)=(1-1 / t) w(t)$. De plus, la fonction composée

$$
x \mapsto w(1 / x)=\frac{u(1 / x)}{1-x}
$$

est strictement décroissante sur $] 0,1[$.

La stricte croissance et la convexité de $v$ sur $[1, \infty$ [, et la stricte convexité de la fonction définie par $x \mapsto 1 / x$ sur ]0,1[, entraînent la stricte convexité de la fonction $x \mapsto v(1 / x)=u(1 / x) / x$ sur $] 0,1[$.

Si la fonction composée $x \mapsto u(x /(1-x))$ est convexe sur son domaine de définition $[1 / 2,1$, alors la fonction intervenant dans (8) est somme d'une fonction strictement convexe et d'une fonction convexe sur $[1 / 2,1$ [; elle est donc strictement convexe sur cet intervalle. Pour vérifier (8)], il suffit de vérifier que sa dérivée à droite au nombre d'or est positive ou nulle. Cela s'écrit

$$
-\frac{1}{\theta^{2}} u(1 / \theta)-\frac{1}{\theta^{3}} u^{\prime}(1 / \theta)+\frac{1}{(1-\theta)^{2}} u^{\prime}\left(\frac{\theta}{1-\theta}\right) \geqslant 0
$$


En tenant compte de l'équation $\theta /(1-\theta)=1 / \theta$, on voit que cette inégalité équivaut à celle de l'énoncé.

Si $u:\left[1, \infty\left[\rightarrow\left[0, \infty\left[\right.\right.\right.\right.$ est strictement convexe, vérifie $u(1)=0$ et $u^{\prime}(1 / \theta) \geqslant u(1 / \theta)$, alors elle vérifie les hypothèses de la proposition 7 . En particulier, c'est le cas des fonctions $t \mapsto(t-1)^{a}$, avec $a>1$ (si $a=1$, la proposition [7]'applique directement). Par ailleurs, les fonctions $t \mapsto \ln ^{a} t$, avec $a \geqslant 1$, sont des exemples de fonctions non convexes vérifiant également cette proposition.

\section{REMERCIEMENTS}

Nous remercions Tanguy Rivoal d'avoir suscité le présent travail, et l'arbitre anonyme d'avoir suggéré de généraliser notre étude. Cette recherche a été rendue possible par le programme Research in pairs du Mathematisches Forschungsinstitut Oberwolfach. Nous remercions cette institution pour les conditions de travail idéales dont nous avons bénéficié.

\section{Références}

[1] M. Balazard et B. Martin - «Comportement local moyen de la fonction de Brjuno », Fund. Math. 218 (2012), p. 193-224.

[2] X. Buff et A. Cheritat - « The Brjuno function continuously estimates the size of quadratic Siegel disks », Ann. of Math. (2) 164 (2006), p. 265-312.

[3] S. Marmi, P. Moussa et J.-C. Yoccoz - « The Brjuno functions and their regularity properties », Comm. Math. Phys. 186 (1997), p. 265-293.

[4] — , «Some properties of real and complex Brjuno functions. », Frontiers in number theory, physics, and geometry I. On random matrices, zeta functions, and dynamical systems, Springer, Berlin, 2006, p. 601-623.

[5] T. RIvoal - «Extremality properties of some Diophantine series », Exp. Math. 19 (2010), p. $481-494$.

[6] J.-C. YocCOZ - Petits diviseurs en dimension 1., Astérisque, vol. 231, Soc. Math. France, Paris, 1995.

BALAZARD, Michel

Aix Marseille Univ, CNRS, Centrale Marseille, I2M, Marseille, France

Adresse électronique : balazard@math.cnrs.fr

MARTIN, Bruno

ULCO, LMPA, Calais, France

Adresse électronique : Bruno.Martin@univ-littoral.fr 